\title{
Physical parameters of the near-Earth asteroids from radar observations
}

\section{Yuri Bondarenko*, Dmitry Marshalov, Yuri Medvedev, Dmitry Vavilov, Maksim Zotov and Andrey Mikhailov}

Institute of Applied Astronomy of the Russian Academy of Sciences, St.Petersburg, Russia

E-mail: bondarernkodiaaras.ru

\begin{abstract}
The Institute of Applied Astronomy in cooperation with the Jet Propulsion Laboratory and Goldstone Deep Space Communications Complex regularly conduct intercontinental bistatic radar observations of near-Earth asteroids using 70-m antenna (DSS-14) to transmit $8560 \mathrm{MHz}(3.5 \mathrm{~cm}$ ) signal and 32-m radio telescopes (RT-32) of "Quasar" VLBI network in Svetloe, Zelenchukskaya and Badary observatories to receive the echoes. To carry out such observations, the existing receiving, conversion and recording systems have been adapted and special software for radar observation scheduling and echo signal data processing has been developed. Since 2015, echoes from 2011 UW158, 2003 TL4, 2003 YT1, 2014 JO25, 2003 BD44, 3122 Florence and 2017 VR12 asteroids have been registered. The continuous wave echo power spectra of these asteroids were obtained and their analysis was carried out. We estimated the size, rotation period, radar albedo and circular polarization ratio of these asteroids.
\end{abstract}

14th European VLBI Network Symposium \& Users Meeting (EVN 2018)

8-11 October 2018

Granada, Spain

${ }^{*}$ Speaker. 


\section{Introduction}

Today, radar astronomy is one of the most precise methods to obtain information about the dynamic and physical properties of near-Earth asteroids (NEAs). The Institute of Applied Astronomy (IAA) regularly participates in radar observations using the 32-m radio telescopes (RT-32) in Svetloe, Zelenchukskaya and Badary observatories as a receivers. The transmitter is the 70-m antenna (DSS-14) of the Goldstone Deep Space Communications Complex. To conduct radar observations of NEAs we adapted the existing receiving, conversion and recording systems of the RT-32 radio telescopes and created a software package for observations scheduling and echo signals processing.

\section{Observations scheduling}

When scheduling radar observations, joint observational windows, the Doppler shift and the Signal-to-Noise ratio (SNR) of the received echo are calculated for a given configuration of transmitting and receiving antenna systems and NEA. The asteroid is specified by Keplerian orbital elements for the selected epoch and physical parameters such as diameter $D$, rotation period $P$ and radar albedo $\hat{\sigma}$. Parameters of the transmitting and receiving antenna systems are their topocentric coordinates, gain, power and frequency of the transmitter signal, noise temperature and the minimum elevation angle of the receiving antenna system. In the calculations, we use DE423 planetary ephemeris, corrections to the Earth's rotation parameters (pole coordinates, precession, nutation, and universal time), and also takes the signal travel time, aberration, gravitational deflection of light, and tropospheric delay into account.

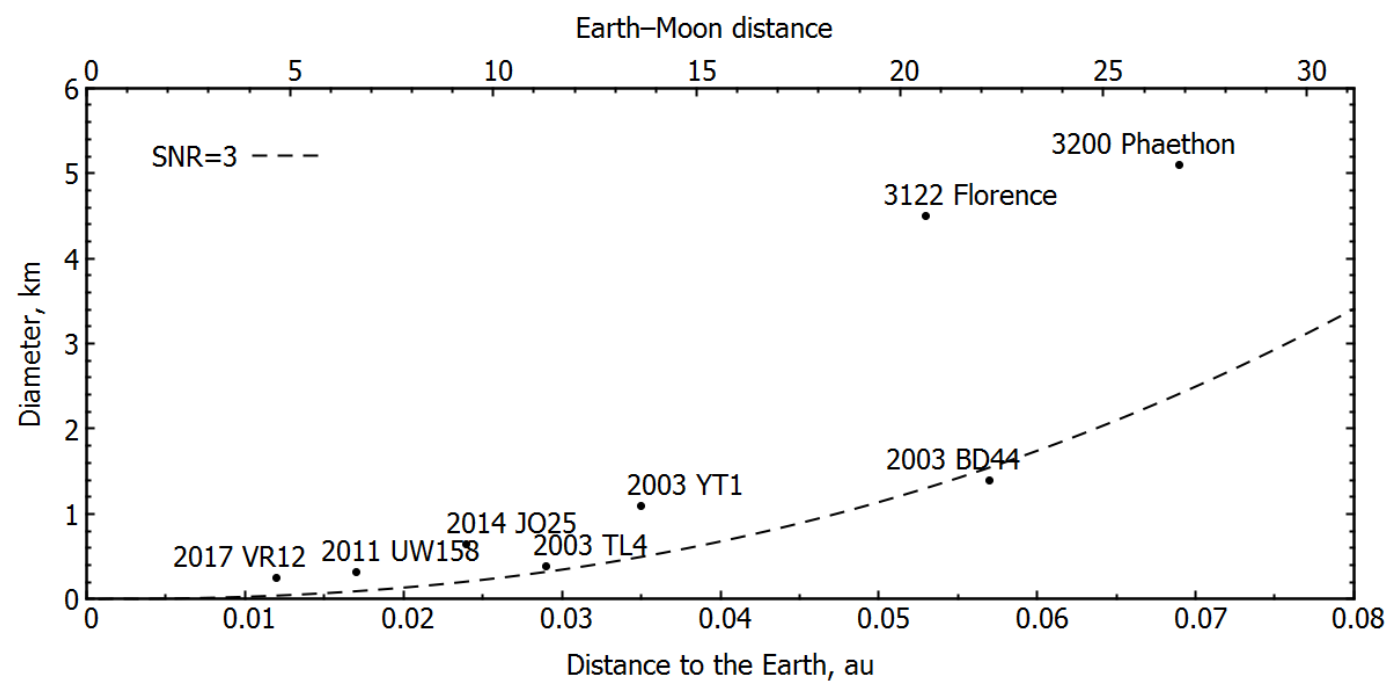

Figure 1: Near-Earth asteroids detected on RT-32.

The Doppler shift of the received echo can vary from tenths to hundreds of hertz per second, which should also be taken into account when choosing the frequency channels of the digital converter on a radio telescope. The decision to conduct radar observations is made on the basis of joint 
with the DSS-14 observational windows, the obtained Doppler shift and SNR. Then, the prepared ephemeris files are uploaded to the radio telescope control system.

Figure 1 shows the size distribution of NEAs detected on RT-32, depending on the distance to the Earth. The dashed line shows the distances to the Earth and the NEAs diameters assuming a Signal-to-Noise Ratio of 3, a rotation period $P=9 \mathrm{~h}$, a radar albedo $\hat{\sigma}=0.1$ and a transmitter power $P_{t x}=400 \mathrm{~kW}$ on a corresponding round-trip light-times. We can consider the obtained curve as a lower limit of the RT-32 sensitivity in bistatic radar observations of NEAs [1].

\section{Observations and data processing}

Usually the DSS-14 radar transmits $150-400 \mathrm{~kW}$ circularly polarized continuous wave (CW) signal at $8560 \mathrm{MHz}(3.5 \mathrm{~cm})$. We use two sets of separate channels at the RT-32 telescopes to receive echoes in the same (SC) and opposite (OC) circular polarizations as that of the transmitted wave. Circular polarization of the signal is reversed after reflection from the plane surface and the maximum power of the reflected signal is expected in the OC polarization, though some of the signal, due to secondary reflections, is received with the same polarization. The received echo is sampled by R1002M Data Acquisition System [2] and recorded by Mark5B. Taking into account the Doppler frequency as a function of time we apply the Fourier transform to the echo time series. As a result we obtain $\mathrm{CW}$ echo power spectra for selected time intervals with the required frequency resolution.

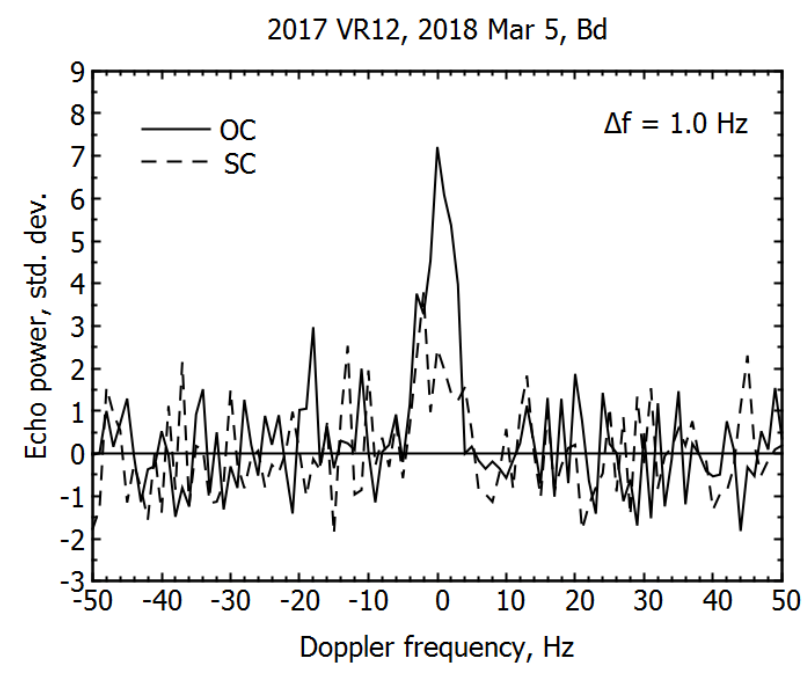

Figure 2: Continuous wave echo power spectra of 2017 VR12. The solid and dashed lines are observed OC and SC spectra, respectively. Each curve has a frequency resolution of $1 \mathrm{~Hz}$.

\section{Analysis of spectra}

From the obtained echo power spectra, we can estimate the Doppler broadening $B$ and the echo's SNR. The CW power spectrum bandwidth as function of time can be used for obtain the 
rotation period $P$ in case of long observation series. The Doppler broadening $B$ is a function of the asteroid's rotation period $P$ and diameter $D$, radar wavelength $\lambda$, and angle between the observer line-of-sight and the asteroid's apparent equator $\delta: B=4 \pi D \cos \delta / \lambda P$. Knowing the $P, B$ and assuming $\delta=0^{\circ}$ we may estimate the lower bound on the asteroid's maximum poleon breadth $D_{\max }$. Taking the geometric relation between echo power spectrum and the shape of rotating asteroid into account, we estimate the hull of asteroids polar silhouette [3].

Circular polarization ratio $\mu_{C}=\sigma_{S C} / \sigma_{O C}$ is the ratio of the integrated SC and OC signal, where $\sigma$ is the radar cross section. A higher ratio indicates a greater degree of near-surface wavelengthscale roughness or multiple scattering. Using estimates of asteroid's diameter and SNR we calculate the radar albedo $\hat{\sigma}$, i.e., the radar reflectivity of the surface.

As an example, at the Figure 2 you may see the OC and SC continuous wave echo power spectra of asteroid 2017 VR12 obtained at Badary observatory on March 5, 2018 from 4:16 to 4:25 UT. Echo power is plotted in standard deviations versus Doppler frequency relative to the estimated frequency of echoes from the asteroid's center of mass. We obtained the Doppler broadening $B=10 \pm 1 \mathrm{~Hz}$ of the spectra and knowing the rotation period $P=1.378 \mathrm{~h}$ found the lower bound on the maximum pole-on breadth $D_{\max }=138 \mathrm{~m}$ at the corresponding rotation phase. Circular polarization ratio of $0.34 \pm 0.01$, indicates that the near-surface of 2017 VR12 at decimeter scales is morphologically rougher than those of most radar-detected NEAs [4]. By integrating the CW spectra we obtained the radar albedo of $0.31 \pm 0.04$ suggests $\mathrm{S}$ or M-class NEA with a bright surface [5].

\section{Acknowledgments}

We thank L. A. Benner, S. Naidu, M. Brozovic and the technical staff at Goldstone for the help with the radar observations. This work was supported by the Russian Scientific Foundation grant No. 16-12-00071.

\section{References}

[1] Marshalova D.A. et.al., Complex for Carrying Out Radar Observations of Near-Earth Objects, Instruments and Experimental Techniques, Vol. 61, No. 4, pp. 577-582, (2018).

[2] Grenkov S.A., Nosov E.V., Fedotov L.V. and Kol'tsov N.E., A Digital Radio Interferometric Data Acquisition System, Instruments and Experimental Techniques, Vol. 53, No. 5, pp. 675-681, (2010).

[3] Ipatov A.V., Bondarenko Y.S., Medvedev Y.D., Mishina N.A., Marshalov D.A., Benner L.A., Radar Observations of the Asteroid 2011 UW158, Astronomy Letters, Vol. 42, Issue 12, pp. 850-855, (2016).

[4] Margi, C. et.al., A radar survey of main-belt asteroids: Arecibo observations of 55 objects during 1999-2003, Icarus, Vol. 186, pp. 126-151, (2007).

[5] Benner, L. et.al., Near-Earth asteroid surface roughness depends on compositional class, Icarus, Vol. 198, pp. 294-304, (2008). 